\title{
ReForma LABORAL LEY No 20.940 BAJO EL PRISMA DE LA TEORÍA DE LAS RELACIONES LABORALES
}

[Labor Reform Law No. 20,940 under the prism of labor relations theory]

\section{Pablo Arellano Ortiz*}

\section{RESUMEN}

El presente trabajo pretende dar cuenta si la reforma laboral de la ley No 20.940 y las regulaciones que ésta introduce a la normativa del derecho colectivo chileno se encuentra en línea con las nociones de la teoría de las relaciones laborales. Para poder realizar el objetivo propuesto de verificación este trabajo se enfoca primero a describir la teoría de las relaciones laborales, revisando algunas definiciones y algunos de sus principales ideólogos. En un segundo momento se procederá a revisar si la normativa actual concuerda con los presupuestos de la teoría ya indicada. Finalmente, señalaremos algunas reflexiones acerca

\section{ABSTRACT}

This paper aims to explain if the labor reform of Law No. 20,940 and the regulations that it introduces to the norms of Chilean collective law is in line with the notions of the theory of labor relations. In order to achieve the proposed verification objective, this paper focuses first on describing the theory of labor relations, reviewing some definitions and some of its main ideologists. In a second moment it will proceed to review if the current regulation agrees with the presuppositions of the already indicated theory. Finally, it will point out some reflections about the compliance in our legislation with the model of labor

ReCiBido el 7 de abril de 2017 y APROBADO el 15 de mayo de 2017

* Doctor en Derecho por la Université de París Ouest Nanterre La Défense, Francia, profesor de Derecho del Trabajo y Seguridad Social, Pontificia Universidad Católica de Valparaíso. Correo electrónico: pablo.arellano@pucv.cl. 
del cumplimiento en nuestra legislación del modelo de las relaciones laborales. Nos interesa entonces verificar que las premisas básicas de teoría de las relaciones laborales se cumplen en nuestro ordenamiento jurídico luego de la reforma citada, tratando de realizar un estudio bajo una óptica distinta.

\section{Palabras claves}

Relaciones laborales - Libertad sindical - Reforma laboral - Sindicato - Negociación colectiva. relations. We are interested then to verify that the basic premises of labor relations theory are fulfilled in our legal order after the reform cited, trying to carry out a study under a different optic.

KEYWORDS

Labour relations - Freedom of association - Labour reform - Union Collective bargaining.

\section{INTRODUCCIÓN}

La reforma a las relaciones laborales realizada mediante la ley No 20.940 es sin lugar a dudas de las más polémicas que se han realizado en los últimos años. Esta tiene su origen en un compromiso político de la campaña de la presidenta Michelle Bachelet, compromiso que es reafirmado en el discurso de 21 de mayo del año 2014". Siendo así parte de las grandes reformas transformadoras del "modelo chileno" que se propuso dicha administración realizar.

${ }^{1}$ En el discurso se hace una alusión expresa a la agenda laboral modernizadora de las relaciones laborales y a los pilares de la libertad sindical, así se señala: "El propósito del programa es realizar importantes cambios a la legislación y a la institucionalidad laboral, creando condiciones para superar las grandes inequidades que aún subsisten en el mercado de trabajo y las grandes dificultades que existen para el ejercicio de los derechos laborales, tanto individuales como colectivos. Nivelar la cancha en materia laboral entre empleador y trabajador es un imperativo de justicia, en la medida que gran parte de la desigualdad se construye en el mercado de trabajo; es decir, en la distribución primaria del ingreso. Buscamos revalorizar el rol del trabajo para enfrentar la desigualdad. Avanzar en esta materia es fundamental para construir un Chile más justo, consistente con la concepción del trabajador y el respeto a su dignidad y derechos. En ese marco, se implementarán políticas en diversas áreas que pueden agruparse básicamente en tres dimensiones: negociación colectiva y fortalecimiento de la actividad sindical; fomento a la participación laboral, y capacitación para el desarrollo y seguro de cesantía. Los desafíos en materia de derechos colectivos afectan a los tres pilares de la libertad sindical: sindicalismo, negociación colectiva y huelga. Por ello, se busca fortalecer al sindicato como sujeto de la negociación colectiva, lo que implica titularidad sindical en la negociación y regula la extensión de los beneficios de la negociación colectiva al sindicato". Disponible en: http://21demayo. gob.cl/2014/pdf/2014 sectorial ministerio-trabajo-y-prevision-social.pdf. 
En este sentido la reforma indicada en la cuenta pública de 21 mayo se encuentra basada en la denominada Agenda Laboral ${ }^{2}$, la cual contiene las bases programáticas del actuar en materia laboral. Dentro de los contenidos de esta agenda se estableció la intención de realizar una reforma a las relaciones laborales teniendo en cuenta dos objetivos principales ${ }^{3}$ : i) ampliar y mejorar la calidad de la negociación colectiva, promoviendo relaciones laborales basadas en el diálogo, la cooperación y el equilibrio entre las partes; ii) fortalecer la participación sindical, promoviendo un movimiento sindical representativo y de calidad que contribuya al desarrollo del país, de las empresas y de los trabajadores y trabajadoras.

Las intenciones de reforma manifestadas en la agenda laboral y en el discurso citado se materializan en un proyecto que fue presentado al Congreso y que se puede identificar por el Boletín $\mathrm{N}^{\circ} 9835-13$, ingresado a tramitación el 29 de diciembre de 2014. Su tramitación fue extensa y a ratos dificultosa en el Congreso, terminando luego de una Comisión Mixta el día 7 de abril de 2016 al oficiarse al ejecutivo el texto aprobado.

En su tramitación legislativa algunos puntos fueron objeto de modificaciones importantes con una serie de indicaciones que intentaron crear un consenso en algunos temas como el remplazo en la huelga o el de la titularidad sindical. No obstante lo anterior, a todas estas dificultades, se debe sumar dos requerimientos parlamentarios ante el Tribunal Constitucional, que fueron acogidos parcialmente ${ }^{4}$, $y$ un veto supresivo presidencial. Finalmente, el proyecto logra transformarse en la ley No 20.940, siendo publicado en el diario oficial el 8 de septiembre de 2016, entrenado en vigencia una parte importante de su articulado el día 1 de abril de $2017^{5}$.

El proyecto fue presentado como una gran reforma al sistema de relaciones laborales chileno. Indicándose que se cambiaría el paradigma existente. Se trataba de "nivelar la cancha"6 atendiendo a la asimetría de poder existente entre empleadores y trabajadores. Sin embargo, una vez avanzada la tramitación se criticó su contenido por no ser en realidad una gran transformación ${ }^{7}$. El desarrollo de la tramitación del proyecto de ley fue bastante discutido tanto por la forma en que se tramitó así como

${ }^{2}$ Ver http://www.agendalaboral.gob.cl

${ }^{3}$ http://www.agendalaboral.gob.cl/las-propuestas/

${ }^{4}$ Los requerimientos interpuestos son los roles No 3016, 3026, 3117, y 3118.

5 Ver disposiciones transitorias de la ley No 20.940. Algunas de éstas prevén la entrada en vigencia con anterioridad para efectos de la calificación de los servicios mínimos y equipos de emergencia, así por ejemplo el artículo tercero transitorio.

${ }^{6}$ Tal como se señaló en el discurso presidencial del 21 de mayo de 2014. laboral/

http://www.elmostrador.cl/noticias/opinion/2015/01/11/la-gran-reformita- 
también por las diversas posturas que adoptaron el Gobierno, los diversos partidos políticos, los actores sociales (empleadores y sindicatos) así como también la opinión pública.

Si bien el contenido ${ }^{8}$ de la ley está centrado en la supresión del libro IV del Código del Trabajo, reemplazándolo por un nuevo texto, también se realizan importantes modificaciones a otras materias principalmente a la regulación de los sindicatos. Algo novedoso dentro de la reforma es la inclusión de normas de genero a favor de las mujeres en la regulación de sindicatos y de la negociación colectiva ${ }^{9}$, se regula el derecho de información ${ }^{10} \mathrm{y}$ se incluye pactos de adaptabilidad ${ }^{11}$, entre otras materias a destacar. Además, se incorpora una nueva institucionalidad: el Consejo Superior Laboral y el Fondo de Formación Sindical y Relaciones Laborales Colaborativas $^{12}$. Cabe indicar que fuera del ámbito de la libertad sindical se realiza una modificación, a nuestro criterio, relevante de los criterios de discriminación establecidos en el artículo 2 del Código del Trabajo. Los temas tratados por esta reforma son muy variados, centrados en la

8 Sobre el contenido de la reforma revisar: Arellano Ortiz, Pablo - Liendo RoA, Ricardo - Walker Errázuriz, Francisco, Reforma Laboral. Ley No 20.940: Moderniza las relaciones laborales (Santiago, Librotecnia, 2016); y ARELLANO OrTiZ Pablo - Severin Concha, Juan Pablo - Feres Nazarala, María Ester (editores) Reforma al Derecho Colectivo del Trabajo, Examen crítico de la ley No 20.940 (Santiago, Thomson Reuters, 2016).

${ }^{9}$ Véase Marzi Muñoz, Daniela, Perspectiva de género, cuota en las directivas y nuevos objetos de negociación: un camino lento hacia el poder, en Arellano OrTiz, Pablo, et al., cit. (n. 9), pp. 63 - 86. Sobre lo mismo, véase Dictamen de la Dirección del Trabajo ORD. $N^{\circ} 1413 / 32,31$-mar-2017. Informa respecto al sentido y alcance de la Ley $N^{o} 20.940$ publicada en el Diario Oficial del 08.09.2016, en particular, en lo referido a las organizaciones sindicales; y Dictamen de la Dirección del Trabajo ORD. $N^{\circ} 1306 / 31,22-m a r-2017$. Informa respecto al sentido y alcance de la Ley $N^{o}$ 20.940 publicada en el Diario Oficial del 08.09.2016, en particular, en lo referido a las disposiciones con perspectiva de género, y su implicancia en los procedimientos para la integración del directorio sindical y de la comisión negociadora.

${ }^{10}$ Véase Palomo Veliz, Rodrigo, El nuevo derecho de información de los sindicatos. Un ejemplo de reforma en la periferia del modelo, en ArELLANO ORTIZ, Pablo, et al., cit. (n. 9), pp. $109-133$.

${ }^{11}$ Véase Rojas MiÑo, Irene, Los pactos de adaptabilidad y el debate sobre su incorporación al sistema jurídico de relaciones laborales chileno, en ARELLANO ORTIZ, et al. cit. (n. 9), pp. $195-211$.

12 Sobre estas materias, véase Arellano Ortiz, Pablo et al., cit. (n. 9). Para su entrada en vigencia se han adoptado los siguientes textos: Decreto $N^{o} 7$, de 03 de marzo de 2017, del Ministerio del Trabajo y Previsión Social: Aprueba Reglamento del Fondo de Formación Sindical y Relaciones Laborales Colaborativas, y Decreto No 6, de 03 de marzo de 2017, del Ministerio del Trabajo y Previsión Social: Aprueba Reglamento del Consejo Superior Laboral. 
negociación colectiva, pero modificando sustancialmente la regulación vigente, aunque sin alterar el modelo ${ }^{13}$.

Todas las materias o reformas introducidas por la ley se hacen bajo el supuesto de las relaciones laborales. De hecho, el nombre de la ley así lo indica. Se trata de una ley que moderniza las relaciones laborales. Esto nos llama particularmente la atención ya que históricamente en nuestro país el estudio de los fenómenos laborales se ha hecho desde la perspectiva del derecho del trabajo y no de las relaciones laborales. En este sentido se puede considerar como un texto con la óptica del derecho del trabajo del Manual de los Profesores William Thayer y Patricio Novoa en sus diversas ediciones ${ }^{14}$. En cuento a las relaciones laborales, este enfoque fue desarrollado en los años 1950 por los académicos de la Universidad de Chile que fundan el Instituto de Organización y Administración de la Universidad de Chile (INSORA). Este instituto en los años 1970 se transforma en un Departamento y pasa a llamarse Departamento de Relaciones del Trabajo y la Organización (DERTO) al interior de la Facultad de Ciencias Económicas y Administrativas (FACEA) en los inicios de 1980 va a dejar de ser un departamento y pasará nuevamente a ser un Instituto, para luego en 1986 ser absorbido por el Departamento de Administración de la misma facultad ${ }^{15}$. Actualmente estudios y desarrollos desde esta perspectiva se

13 Sobre el mantenimiento del modelo de relaciones, véase CAAMAÑo Rojo, Eduardo, Crítica a las ideas fundantes del actual modelo normativo de negociación colectiva en Chile y a su reforma, en Revista de Derecho de la Pontificia Universidad Católica de Valparaíso XLVI (2016) pp. 381 - 406; Gamonal ConTreras, Sergio, La reforma laboral: interpretación, antinomias y vacios, en Revista Laboral Chilena (2016) pp. 73-78; Gamonal Contreras, Sergio, La reforma laboral: un paso adelante y dos atrás, en Revista Laboral Chilena (2015), pp. 67-70; NARBOna, Karina, Antecedentes del modelo de relaciones laborales chileno (2a edición, Santiago, Fundación Sol, 2015).

${ }^{14}$ Thayer Arteaga, William y Novoa Fuenzalida, Patricio, Manual de Derecho del Trabajo (T. I, 2007; T. II, 2008; T. III, 2008 y T. IV 2010, Editorial Jurídica de Chile). A este respecto se debe indicar que se cita a estos autores porque, en general, se les considera como el manual de base para muchos programas de Derecho del trabajo. Sin embargo, se debe indicar que en general la doctrina chilena se ha ocupado de los fenómenos laborales bajo la óptica de derecho del trabajo y seguridad social.

${ }^{15}$ De ello da cuenta por ejemplo la publicación de Walker Errázuriz, Francisco, y Muga Naredo, José Antonio, Las relaciones industriales (Santiago, Universidad de Chile - DERTO, 1978), pp. 44. Además, se encuentran rastros de las investigaciones realizadas tanto por el INSORA en revistas extranjeras, como, por ejemplo, DAvIs, Stanley M., The Politics of Organizational Underdevelopment: Chile, en Industrial and Labour Relations Review, vol. 24, No. 1 (1970), pp. 73-83; y LANDSBERGER, Henry A.; Barrera, Manuel y Toro, Abel, The Chilean Labor Union Leader: A Pre- 
pueden encontrar en el Centro de Relaciones Laborales, Trabajo y Diálogo Social o Centro de Relaciones Laborales de la Universidad Central ${ }^{16}$.

Observamos que las relaciones laborales no consisten en una aproximación puramente jurídica a los fenómenos y problemáticas del trabajo, es una óptica más completa y compleja del estudio de dichos objetos. El presente trabajo pretende evidenciar si la reforma laboral de la ley $\mathrm{N}^{\circ}$ 20.940 y las regulaciones que ésta introduce a la normativa del derecho colectivo chileno se encuentra en línea con las nociones de la teoría de las relaciones laborales. Nos interesa entonces verificar que las premisas básicas de teoría de las relaciones laborales se cumplen en nuestro ordenamiento jurídico luego de la reforma citada.

Para poder realizar el objetivo propuesto de verificación este trabajo se enfocará primero a describir la teoría de las relaciones laborales, revisando algunas definiciones y algunos de sus principales ideólogos. En un segundo momento se procederá a revisar si la normativa actual concuerda con los presupuestos de la teoría ya indicada. Finalmente, señalaremos algunas reflexiones acerca del cumplimiento en nuestra legislación del modelo de las relaciones laborales.

\section{TeOría de las RElaciones laborales COMO MARCO DE ESTUDIO DE LOS FENÓMENOS LABORALES}

En primer término, analizaremos la a ratos confusión terminológica sobre el nombre de esta teoría, existiendo diferentes acepciones para esta manera de estudiar los fenómenos laborales. En este sentido cabe indicar que "el concepto relaciones laborales presenta aspectos compartidos pero diferentes con otras nociones que se emplean en este ámbito. El concepto anglosajón de "relaciones industriales" prima el aspecto colectivo de las relaciones laborales (abandono de la perspectiva individual); en la tradición europea también se diferencia entre "relaciones salariales", para referirse al trabajo asalariado, y "relaciones profesionales", relativas a toda forma de trabajo mercantil. También coincidiría parcialmente con el concepto de "relaciones de empleo", al menos en cuanto a un objeto que no se ceñiría el trabajo asalariado, pero con la diferencia de que el enfoque de este concepto

liminary Report on His Background and Attitudes, en Industrial and Labour Relations Review, vol. 17, No. 3 (1964), pp. 399-420.

$16 \mathrm{Se}$ sugiere revisar su sitio web http://www.ucentral.cl/centro-deestudios-y-asesorias-en-relaciones-laborales-trabajo-y/prontus ucentral2012/2012-01-02/183557.html Cabe destacar que dicha casa de estudios imparte una maestría bajo este enfoque, el que se Maestría Internacional sobre Políticas en Trabajo y Relaciones Laborales y su última versión comenzó el año 2015. 
vendría referido a la colocación (la ocupación y su gestión). El concepto de "relaciones laborales" tampoco coincide con el de "recursos humanos", disciplina centrada en las políticas de organización y gestión del trabajo en la empresa (por tanto, marginalizante de la dimensión colectiva del fenómeno a niveles supraempresariales)"17. Cabe indicar, además, que la noción de Relaciones Industriales ha estado tradicionalmente íntimamente ligada a la relación entre los sindicatos y las empresas, en asuntos del ámbito de la relación colectiva. De esta manera, los convenios y conflictos colectivos, han sido los caballos de batalla de este campo del quehacer de la relación entre los sindicatos y la gerencia empresarial, en los espacios productivos, como señala el profesor Lucena ${ }^{18}$.

Debemos señalar que se ha indicado que "la expresión "relaciones laborales" se utiliza para expresar un tipo de relación social de producción, reproducción y distribución surgida en el siglo XIX. En un plano más descriptivo o socioeconómico, la noción se referiría a la suma de interacciones, formales e informales, entre tres sujetos o actores colectivos histórica y socialmente muy característicos, los trabajadores ("clase obrera"), los empresarios ("clase burguesa"), el Estado ("poder constitucionalmente establecido"), y que consisten en obligaciones, derechos y poderes contraídos entre ellos en torno a la prestación de una forma de trabajo (el trabajo asalariado). En un plano más institucional o jurídico, por relaciones laborales puede entenderse básicamente a una forma concreta de regulación y ordenación de la forma mercantil de actividad propia del trabaja asalariado y que comprende el conjunto - o sistema- de instituciones, normas y prácticas que estructuran las interacciones entre las personas asalariadas y los empresarios-empleadores, y de éstos con el Estado, en una amplia gama de ámbitos sociopolíticos y económicos" 19 .

Por su parte, Kaufman señala que en el desarrollo de una base única para la teoría de las relaciones industriales, con su cuerpo asociado de herramientas y conceptos, la constatación es que ha sido variado, pero a fin de cuentas sin duda de manera decepcionante. Esto porque, como señala este autor, a pesar del siglo de esfuerzos, así como numerosos libros y artículos

${ }^{17}$ Monereo Pérez, José Luis - Moreno Vida, María Nieves - Márquez Prieto, Antonio - Almendros González, Miguel Ángel; Díaz Aznarte, María Teresa; Fernández Avilés, José Antonio - Salas Porras, María, Manual de teoría de las relaciones laborales (Madrid, Tecnos, 2011) p. 23.

${ }^{18}$ LuCENA, Héctor, El enfoque de las relaciones industriales y los estudios laborales, en Enrique de la Garza Toledo (comp.), Los retos teóricos de los estudios del trabajo hacia el siglo XXI (Buenos Aires, Consejo Latinoamericano de Ciencias Sociales, 1999), p. 89.

${ }^{19}$ Monereo Pérez, José Luis et al., cit. (n. 18) p. 22. 
sobre el tema de la teoría de las relaciones industriales, hay relativamente poco impacto o presencia de esta teorización en la literatura de las últimas dos décadas ${ }^{20}$. Podemos afirmar que sobre las relaciones industriales existe una gran teorización y desarrollo académico principalmente en Estados Unidos de Norteamérica y en Inglaterra durante la primera mitad de 1900, pero que luego ha ido decayendo su estudio.

De hecho, para la doctrina norteamericana se indica como fecha de nacimiento del estudio de las relaciones laborales el año 1920, en el mismo año se funda la Industrial Relations Association of America (IRAA) ${ }^{21}$. En dicho país se considera como un hito relevante el establecimiento del Industrial and Labour Relations Review en el año $1947^{22}$, el cual desde entonces ha publicado a los principales exponentes de la academia de dicho país. Sin embargo, desarrollos sobre esta área de estudio se pueden encontrar en el periodo inmediatamente anterior, como veremos más adelante.

Debemos constatar que ha existido una evolución terminológica, desde la idea de las relaciones industrial a lo que hoy día se indica como relaciones laborales ${ }^{23}$.

Teniendo en cuenta las concepciones más recientes de esta manera de estudio de Monereo Perez y otros indican que las relaciones laborales se articulan, estructural y funcionalmente, en torno a tres dimensiones básicas estrechamente interconectadas ${ }^{24}:$ i) como interacciones de intercambio económico que presuponen una dimensión formal contractual. Principalmente a través del contrato de trabajo; ii) las relaciones laborales tienen una dimensión sociopolítica en la medida que constituyen relaciones de poder entre personas y colectivos con intereses contrapuestos, y con desigual poder socioeconómico; iii) las relaciones presentan una dimensión normativa - institucional, pues constituyen una relación de ordenación social de una esfera de la vida social, a través de un sistema plural regulador (integrado por normas estatales y colectivas), y una institucionalización de los actores del sistema y de sus modos de actuación.

${ }^{20}$ Kaufman, Bruce, The Theoretical Foundation of Industrial Relations and its Implications for Labor Economics and Human Resource Management, en Industrial \& Labor Relations Review (2010) 64, p. 75.

${ }^{21}$ Kaufman, Bruce, The origins \& evolution of the field of Industrial relations in the United States, en Cornell Studies in Industrial and Labor Relations, 25, (1993), p. 19.

${ }^{22}$ Kaufman, Bruce, The origins \& evolution, cit. (n. 22), p. 71, y Reed Tripp, L. The Industrial Relations Discipline in American Universities, en Industrial and Labour Relations Review, vol. 17, No. 4 (1964), pp. 612-618.

${ }^{23}$ No es objeto de este trabajo despejar las diferencias terminológicas de ambos conceptos. En estas líneas nos interesa sentar la idea que ha existido una evolución conceptual.

${ }^{24}$ Monereo Pérez, José Luis et al., cit. (n. 18) p. 23 y 24 
En cuanto a las diferentes perspectivas de análisis bajo la óptica de las relaciones laborales Monereo Pérez et $a .^{25}$ señalan que las diversas teorías de las relaciones laborales se mueven dentro de las siguientes líneas o ejes contrales: una primera que trata de explicar la cuestión del orden, las relaciones de conflicto y consenso en las relaciones laborales. El debate sobre estas cuestiones ha tenido especial relevancia durante el proceso de industrialización y desarrollo del capitalismo, en la búsqueda de una respuesta sobre el funcionamiento del orden socioeconómico. Una segunda línea viene marcada por el debate teórico sobre las relaciones entre instituciones sociales, cohesión social y mercado laboral. En ella se discute el papel de la economía y del mercado en el seno de la sociedad.

$\mathrm{Al}$ momento de clasificar las diversas visiones ${ }^{26}$ cerca de las relaciones laborales se puede optar por una clasificación como la recién presentada. Sin embargo, debemos advertir que cada propuesta que se ha efectuado responde a un contexto cultural e histórico diferente y propio de cada país. Los estudios comparados de relaciones laborales resultan particularmente complejos debido a las diferentes concepciones del trabajo que se puede encontrar. Así Richard Hyman señala en que los estudios comparados entre países bajo estas ideas son cuestionables por dos motivos: en primer lugar, porque la "composición química" del mundo social es mucho más compleja que en el caso del mundo natural. En segundo lugar, porque es necesario preguntar: ¿qué es una variable, y qué es una constante? Esto debido a poder fijar puntos de comparación debido a que se generan muchos problemas de traducción comparando institutos que tienen el mismo nombre en sentido literal pero que en realidad no lo $\operatorname{son}^{27}$.

Cabe agregar que la complejidad del estudio de los fenómenos laborales es concebida de distintos ángulos ${ }^{28}$. Algunos culpan a que la legislación se ha tornado compleja y otros a la naturaleza propia del objeto de estudio.

No obstante, las dificultades indicadas para el estudio de las relaciones laborales, quisiéramos detenernos brevemente en dos de las posturas que

${ }^{25}$ Aquí se ha hecho un resumen de lo expuesto en Monereo Pérez, José Luis et al., cit. (n. 18) p. 25 ss.

${ }^{26}$ Una revisión sintética de éstas visiones se puede encontrar en DERBER, Milton, Divergent tendencies in industrial relations research, en Industrial and Labour Relations Review, vol. 17, No. 4 (1964) pp. 598-611.

${ }^{27}$ Hyman, Richard, How Can We Study Industrial Relations Comparatively?, en Blanpain, Roger - Bromwich, William - Rymkevich, Olga - Spattini, Silvia (eds) Bulletin of Comparative Labour Relations, vol. 70, The Modernization of Labour Law and Industrial Relations in a Comparative Perspective (Kluwer, 2009), p. 5.

${ }_{28} \mathrm{Al}$ respecto, LoKIEC, Pascal, Il faut sauver le droit du travail! (Paris, Odile Jacob, 2015) y Badinter Robert y Lyon-CAEn, Antoine, Le travail et la loi (Paris, Fayard, 2015). 
se podrían considerar como clásicas sobre el estudio de los fenómenos laborales. La primera de ellas dice relación con los estudios realizados por los Webbs, por su carácter inicial e histórico, y la segunda es la desarrollada por John T. Dunlop, debido a su amplia acogida de sus ideas en la poca doctrina chilena de relaciones laborales de la segunda mitad del siglo XX ${ }^{29}$.

La primera de las posturas que queremos analizar ha sido encasillada como aquella que se ocupa de las relaciones laborales desde un enfoque histórico ${ }^{30}$, principalmente por sus trabajos en ese sentido ${ }^{31}$, está a cargo de un matrimonio inglés de precursores en los estudios de los fenómenos laborales, Sidney Webb y Beatrice Potter, quienes se casaron en 1892, han sido uno de los equipos de investigación marido-mujer más productivos e influyentes en la historia de las ciencias sociales ${ }^{32}$. Entre las decenas de libros y artículos que escribieron durante una carrera de seis décadas, el volumen de más de 900 páginas Industrial Democracy $(1897)^{33}$ se destaca como su obra de mayor impacto. Dicho libro es considerado como un ejemplo del enfoque institucional temprano en la economía política, en él se realiza una exhaustiva investigación de campo con un enfoque inductivo I empirista del desarrollo teórico, un cuerpo de la teoría que integra de manera literaria ideas de diversos escritores y disciplinas de su época. Se incluye además un programa progresivo de políticas públicas enfatizando el control social y la reingeniería de las instituciones para producir resultados más eficientes y equitativos ${ }^{34}$. Lamentablemente se constata que existe una modesta literatura sobre la biografía y pensamiento político de los $\mathrm{Webbs}^{35}$. El gran aporte de ellos consiste en un estudio de las diversas

${ }^{29}$ Nos referimos aqui a los trabajos del INSORA y del DERTO.

${ }^{30}$ Monereo Pérez, José Luis et al. cit. (n. 18) p. 29.

31 Véase Webв, Sidney y Weвb, Beatrice, History of Trade Unionism (London, Amalgamated Society of Engineers, 1894).

${ }^{32}$ Kaufman, Bruce, Sidney and Beatrice Webb's Institutional Theory of Labor Markets and Wage Determination, Working Paper 2013-1-2, W. J. Usery Workplace Research Group Paper Series, Andrew Young School of Policy Studies, Georgia State University, January 2013, p. 4.

33 Este libro fue publicado originalmente en dos volúmenes y en el año 1902 aparece una nueva edición en un solo volumen: WeBb, Sidney y WeBb, Beatrice, Industrial Democracy (London, Longmans, Green, 1902) ; WebB, Sidney y WebB, Beatrice, Industrial Democracy vol. I (London, Longmans, Green, 1897): WeBB, Sidney y WeвB, Beatrice, Industrial Democracy, vol. II (London, Longmans, Green, 1897)

${ }^{34}$ Kaufman, Bruce, Sidney and Beatrice Webb's, cit. (n. 33) p. 3

35 Como señala Kaufman, Bruce, Sidney and Beatrice Webb's, cit. (n. 33) p. 2. Uno de los estudios relevantes sobre los Webbs es: Cole, Margaret, The Webbs and Social Theory, en The British Journal of Sociology, vol. 12, No. 2 (1961), pp. 93-105. 
teorías económicas surgidas dentro del contexto de la revolución industrial en relación al trabajo y poner en relieve el estado de situación de la industria.

La segunda postura que nos interesa presentar es la desarrollada por el norteamericano John T. Dunlop. Este autor analiza a las relaciones laborales postulando lo que se ha denominado una teoría del "sistema de relaciones industriales" 36 . Para Dunlop "en cualquier instante de su desarrollo se considera un sistema de relaciones industriales a aquel que está compuesto por ciertos actores, ciertos contextos, una ideología que mantiene unido al sistema de relaciones industriales y un cuerpo de normas creadas para dirigir a los actores en el lugar y comunidad de trabajo" 37 . La proposición de Dunlop ha sido ampliamente recogidas en Estados Unidos de Norteamérica y también en nuestro país. Aunque debemos señalar que su impacto se torna más global gracias a un trabajo colectivo sobre "El industrialismo y el hombre industrial" publicado en la década de 1960. Dicho trabajo estudia las relaciones entre la industrialización, los dirigentes y los grupos de trabajadores, en donde se predice que las relaciones se tenderán a uniformizar ${ }^{38}$. Lo que podríamos estimar como una predicción de lo que hoy se entiende por globalización.

Estas posturas que podríamos considerar como clásicas dentro de las relaciones laborales se siguen desarrollando, aunque han aparecido nuevas tendencias ${ }^{39}$ dentro de las cuales nos gustaría destacar las posturas de Richard Hyman ${ }^{40}$, Bruce Kaufman ${ }^{41}$ y Niklas Luhmann ${ }^{42}$.

Las relaciones laborales también han sido estudiadas en la región la-

${ }^{36}$ Esta teoría fue propuesta en Dunlop, John T. Sistema de relaciones industriales (Santiago, Editorial del Pacífico, 1967).

${ }^{37}$ Dunlop, John T., cit. (n. 37) p. 19.

${ }^{38}$ Kerr, Clark; Dunlop, John T. - Harbison, Frederick - Myers, Charles A., Industrialism and Industrial Man (New York, Harvard University Press - Oxford University Press, 1960).

${ }^{39}$ Este trabajo no pretende desarrollar todas las tendencias, tan solo dar cuenta de las posiciones clásicas descritas y de la existencia de trabajaos más recientes.

${ }^{40}$ Ver por ejemplo de este autor: Hyman, Richard, Tres hipoótesis sobre el futuro de las relaciones laborales en Europa, en Revista Internacional del Trabajo, vol. 134 (2015), 1, pp. 5-15; HyMan, Richard, Relaciones industriales: una introducción marxista (Madrid, Blume, 1981); Hyman, Richard, How Can We Study, cit. (n. 28).

${ }^{41}$ Sobre este autor ver KaUfman, Bruce, The global evolution of industrial relations: Events, ideas and the IIRA, (Geneva, International Labour Office, 2004); KAUfMAN, Bruce, (ed.) Theoretical perspectives on work and the employment relationship (Illinois, Industrial Relations Research Association Series, 2004); KaufMan, Bruce, The origins \& evolution, cit. (n. 22); Kaufman, Bruce, The Theoretical Foundation of Industrial Relations, cit (n. 21).

${ }^{42}$ Sobre este autor vése CASTro Sáez, Bernardo A., Aportes de Niklas Lubmann a la teoría de la complejidad, en Polis, Revista de la Universidad Bolivariana, vol. 10, No. 
tinoamericana. Así, encontramos diversas opiniones desde la perspectiva regional. Por ejemplo, Lucena, quien, en referencia a las relaciones laborales, señala que desde fines de los años setenta toda la región experimenta profundas transformaciones en sus sistemas de acumulación, se constata así, los impactos de la internacionalización y la globalización, los que no han dejado a país alguno libre de influencias ${ }^{43}$.

Una observación similar es efectuada por Luis Aparicio Valdez quien estima que "América Latina, de manera desigual pero constante, se está moviendo hacia la integración, con un considerable potencial para aumentar su fortaleza económica. Los principales países, en particular Brasil, pero también Chile, Costa Rica - que está empezando a surgir - y hasta Uruguay y Argentina son altamente competitivos, también en términos de innovación tecnológica y liderazgo productivo. Sin embargo, dentro de estos países existen enormes disparidades en los ingresos y las condiciones sociales. Para una parte considerable de la población las normas de la cuales que hablamos son realmente una cuestión de buenos deseos en lugar de normas mínimas para la defensa. Como resultado de ello hay una necesidad de un debate en dos frentes: el exterior, para avanzar en sintonía con las necesidades de la globalización, e internamente, para avanzar en el ámbito local, con un enfoque equilibrado, a fin de que los habitantes de varios países comiencen a participar en los procesos globales" ${ }^{4}$.

Hemos afirmado que hoy en día "las relaciones laborales atienden a un número cada vez más reducido de trabajadores. La precariedad, la flexibilidad y la informalidad laboral hacen que cada vez más los trabajadores vayan abandonando de jure o de facto el marco protector de las relaciones laborales. Entonces el estudio de las relaciones laborales se hace cada vez más complejo e interdisciplinario" ${ }^{45}$.

Aparicio Valdez y Bernedo Alvarado señalan claramente que "no hay actividad más esencial para la humanidad y para el individuo que el trabajo. Las relaciones laborales son esencialmente interdisciplinarias. Si no fuera

29 (2011), pp. 283-300; Urteaga, Eguzkil, La teoría de sistemas de Niklas Luhmann, en Contrastes. Revista Internacional de Filosofía, vol. XV (2010), pp. 301-317.

${ }^{43}$ LuCENA, Héctor, cit. (n. 19) p. 89.

${ }^{44}$ Aparicio Valdez, Luis y Bernedo Alvarado, Jorge, The Case for the Comparative and Interdisciplinary Study of Labour Relations, en BLANPAIN, Roger - BROMWICH, William et al., cit. (n. 28), p. 31.

45 Walker Errazuriz, Francisco, y Arellano Ortiz, Pablo, Derecho de las relaciones laborales, t. 1. Derecho Individual del Trabajo (Santiago, Librotecnia, 2016) p. 33. 
así, no existirían. Este es el reto y el estímulo para continuar por el camino del estudio comparado y multidisciplinario de las relaciones laborales" ${ }^{\$ 6}$.

\section{LA REFORMA DE LA LEY No 20.940 CONFRONTADA A LAS}

\section{RELACIONES LABORALES}

Caamaño ha señalado, a propósito de la ley No 20.940, que "la reciente aprobación de la reforma laboral puso en evidencia que ésta no fue la tan anhelada oportunidad para reconciliar nuestra legislación laboral con el derecho a la libertad sindical, ajustándola, a lo menos, a las reglas básicas emanadas de los convenios de la OIT que el país ha ratificado. Es más, pareciera que la ansiada libertad sindical está aún demasiado lejos.

Por tanto, sigue latente el desafío de reformular las bases fundantes del sistema de relaciones laborales colectivas y conectarlas con la concreción de un verdadero Estado democrático de Derecho en el país". ${ }^{47}$

Compartimos la evaluación realizada por el autor citado, en el sentido que el modelo de relaciones laborales no ha sido cambiado. Se mantienen las reglas básicas sobre las cuales se estructura la regulación de los fenómenos laborales. Sin embargo, una evaluación bajo el prisma de la teoría de las relaciones laborales no resulta ser muy sencillo.

Como hemos visto en el acápite anterior, el estudio de los fenómenos laborales por la teoría de las relaciones laborales no es una cuestión pacífica. Existe una gran diversidad de posturas y tendencias al respecto, aunque la característica más relevante es que se trata de un estudio que se realiza a través de una mirada multidisciplinaria, es decir, se conjuga derecho, sociología, economía y muchas veces recursos humanos.

Para poder realizar una confrontación de la regulación chilena luego de la reforma de la ley No 20.940 a la teoría de las relaciones laborales, adoptaremos una posición y nos ceñiremos a la idea de sistema de relación laborales propuesta por Dunlop. De esta manera, y como ya lo hemos propuesto $^{48}$, consideraremos que un sistema de relaciones laborales debe tener 3 elementos: actores, una ideología y un aspecto valorativo o axiológico. A continuación, revisaremos el resultado de la reforma de la ley No 20.940 a la luz de éstos tres elementos.

a) Los actores de las relaciones laborales. - Los actores del sistema pueden revestir diversas calidades, así se hable de actores directos y de actores

\footnotetext{
${ }^{46}$ Aparicio Valdez, Luis y Bernedo Alvarado, cit. (n. 35) p. 35

47 Caamaño Rojo, Eduardo, cit. (n. 14) pp. 402 y 403.

${ }^{48}$ Lo hemos propuesto así en Walker Errazuriz, Francisco, y Arellano Ortiz,
} Pablo, cit. (n. 46) p. 26-32. 
indirectos ${ }^{49}$. Ellos son los trabajadores y sus organizaciones, el empleador y sus organizaciones, y el Estado.

En cuanto al Estado como actor del sistema este ha visto retocada sus prerrogativas de manera importante. Por un lado, el rol fiscalizador se mantiene y su intervención como mediador a través de la Dirección del Trabajo se sigue manteniendo. Por otro lado, se regula de una manera diferente la mediación de conflictos laborales y el arbitraje ${ }^{50}$. En este sentido se establece un registro nacional de árbitros, el cual miramos con atención en cuanto a la posibilidad que éstos puedan contribuir efectivamente a la solución de conflictos colectivos.

En cuanto al actor empleador y sus organizaciones, a decir verdad, éstos no se ven mayormente afectados por la nueva normativa. Su forma de organización y de toma de decisiones sigue siendo la misma. Eventualmente, ellos podrían considerarse afectados debido a la incorporación de normas relativas al derecho de información ${ }^{51}$. Sin embargo, estimamos que para un verdadero equilibrio de las relaciones laborales y que contribuyan a un sano dialogo social los sindicatos deben tener acceso a información concreta que les permita poder tomar sus acciones de la manera más informada posible.

Los sindicatos, como actores del sistema, son quienes en principio ven alterada sus facultades y medios de acción dentro del sistema de relaciones laborales chileno. A nuestro parecer hay dos aspectos que resultan de particular importancia. El primero de ello dice relación con la modificación al quorum necesario para poder constituir un sindicato en aquellas empresas que tienen 50 trabajadores o menos de acuerdo a la nueva redacción del artículo 227 del Código del Trabajo. Así, en estas empresas podrían constituir un sindicato ocho trabajadores, siempre que representen como mínimo el 50 \% del total de éstos. Si la empresa tiene un número impar de trabajadores, el porcentaje se calcula sobre el número par inmediatamente anterior a aquel. A este respecto ya hemos señalado nuestra preocupación por esta nueva regla ${ }^{52}$, esto debido a que la exigencia del $50 \%$ de trabajadores para poder formar un sindicato es una regla que había sido eliminada del Código del Trabajo en el año 2001 por la Ley No 19.795. Más aun, no

49 Walker Errazuriz, Francisco, y Arellano Ortiz, Pablo, cit. (n. 46), p. 27.

50 Ver artículos 378 y siguientes del Código del Trabajo.

51 Sobre la manera de ejercicio de este derecho, Dictamen de la Dirección del Trabajo ORD. $N^{\circ} 5935 / 96,13-d i c-2016$. Informa respecto al sentido y alcance de la Ley $N^{\circ} 20.940$ publicada en el Diario Oficial del 08.09.2016, en particular, en lo referido a Derecho a Información; y Dictamen de la Dirección del Trabajo ORD. $N^{\circ} 1415 / 34,31$ mar-2017. Complementa Dictamen 5935/96 de 13.12.2016. Véase también PALOMO Veliz, Rodrigo, cit. (n. 11).

${ }^{52}$ Arellano Ortiz, Pablo et al., cit. (n. 9), p. 44-45. 
entendemos las razones por las cuales se vuelve a incorporar esta exigencia, ya que su eliminación en el año 2001 se realiza para facilitar la formación de sindicatos y dar cumplimiento así al Convenio $\mathrm{N}^{\circ} 87$, de 1948, de la Organización Internacional del Trabajo, ratificado por nuestro país el 1 de febrero de 1999. Ahora se vuelve a incluir bajo la misma premisa. Estimamos, entonces, que el fortalecimiento de los sindicatos mediante la reforma de esta norma no se concretará en la práctica.

Cabe agregar que en aquellas empresas en que no existan sindicato el quorum de 8 trabajadores que representen al $50 \%$ de los trabajadores debe completarse en el plazo de un año. Si transcurrido un año no logra ser alcanzado el porcentaje la personalidad jurídica del sindicato caduca por el solo ministerio de la ley. Lo que tal como se viene señalando puede ser atentatorio a la voluntad de los trabajadores a formar un sindicato, $y$ por ende, vulneratorio de la libertad sindical.

El segundo aspecto en que consideramos que los sindicatos como actores se ven afectados con la ley No 20.940 dice relación con la titularidad sindical. Esta fue cercenada del proyecto de ley mediante sentencia del Tribunal Constitucional ${ }^{53}$ produciendo un desequilibrio importante en el contenido del proyecto. Dejando además algunos pasajes sin regulación alguna en el Código del Trabajo. La idea de regular la titularidad sindical dice relación con darle preeminencia a los sindicatos por sobre los grupos de hecho para la reivindicación de los derechos colectivos. Sin embargo, el resultado presente en el Código del trabajo es que los sindicatos no tienen la iniciativa exclusiva, la titularidad, para la negociación colectiva, ya que ésta puede ser llevada a cabo por lo que se denomina "grupos negociadores", quienes pueden acordar un nuevo tipo de instrumento colectivo: "el acuerdo de grupo negociador". Lamentablemente, se debe constatar que del accidentado trámite legislativo no quedo ninguna definición de grupo negociador ni de acuerdo de grupo negociador. El profesor Gamonal ha denominado a estos grupos como los grupos fantasmas ${ }^{54}$.

Queda entonces la incógnita acerca de cómo negociaran estos grupos. Ello ha motivado la solicitud de pronunciamiento a la Dirección del Trabajo. Quien, respondiendo la solicitud, indica que al no haber texto legal que interpretar la Dirección de Trabajo no puede resolver la cuestión planteada ya que de hacerlo pasaría a legislar ${ }^{55}$.

${ }^{53}$ Ver requerimientos, roles No 3016 y 3026.

${ }^{54}$ Gamonal Contreras, Sergio, La reforma laboral: interpretación, antinomias y vacios, cit. (n. 14) p. 77.

${ }^{55}$ Dictamen de la Dirección del Trabajo ORD. $N^{\circ} 1163 / 29,13-m a r-2017$. Informa respecto al sentido y alcance de la Ley $N^{\circ} 20.940$ publicada en el Diario Oficial del 08.09.2016, en particular, atiende consulta sobre grupos negociadores. 
La Comisión de Expertos en Aplicación de Convenio y Recomendaciones de la Organización Internacional del Trabajo a principios del 2017 le ha recordado a nuestro país en relación a los grupos negociadores "que los grupos de trabajadores sólo deberían poder negociar convenios o contratos colectivos en ausencia de tales organizaciones. Adicionalmente, se ha constatado en la práctica que la negociación de las condiciones de trabajo y empleo por medio de grupos que no reúnen las garantías para ser considerados organizaciones de trabajadores puede ser utilizada para desalentar el ejercicio de la libertad sindical y debilitar la existencia de organizaciones de trabajadores en capacidad de defender de forma autónoma los intereses de los trabajadores durante la negociación colectiva" ${ }^{56}$. La Comisión claramente no se encuentra satisfecha con la manera como se ha tratado en nuestra país a los grupos negociadores y le "pide al Gobierno que busque, a través del diálogo social, soluciones que reconozcan el papel fundamental y las prerrogativas de las organizaciones representativas de trabajadores y sus representantes y que prevean mecanismos para evitar que la intervención de un grupo negociador en la negociación colectiva en ausencia de sindicato pueda debilitar la función de las organizaciones de trabajadores o socavar el ejercicio de la libertad sindical"57.

Estimamos que en este elemento "el debilitamiento de la actividad sindical y el bloqueo a la noción de titularidad sindical harán que se limite el desarrollo de la negociación colectiva" ${ }^{58}$. En lugar de fortalecer al sindicato y a su actividad se producirá el efecto contrario.

b) La ideología del sistema de relaciones laborales. - Este elemento dice relación con las ideas matrices que inspiran el sistema de relaciones laborales en un país determinado ${ }^{59}$.

Esta es la principal motivación para la realización de este trabajo. El título de la ley No 20.940 es “moderniza las relaciones laborales” y entonces ello debería implicar una concepción en particular. Lamentablemente se aprecia que el contexto ideológico de la nueva redacción del Libro IV, principal modificación al Código del Trabajo, mantiene los presupuestos ya

${ }^{56}$ Organización Internacional del Trabajo, Informe de la Comisión de Expertos en Aplicación de Convenios y Recomendaciones (artículos 19, 22, 23 y 35 de la Constitución), Tercer punto del orden del día: Informaciones y memorias sobre la aplicación de convenios y recomendaciones, Informe III (Parte 1A), Informe Generaly observaciones referidas a ciertos países Conferencia Internacional del Trabajo, 106.a reunión, 2017 (Ginebra, Oficina Internacional del Trabajo, 2017), p. 88.

57 Organización Internacional del Trabajo, cit. (n. 57), p. 88

58 Arellano Ortiz, Pablo et al., cit. (n. 9), p. 229.

59 Walker Errazuriz, Francisco, y Arellano Ortiz, Pablo, cit. (n. 46) p. 27 28. 
establecidos en el Plan Laboral y en el Código del trabajo de 1987. Desde ese entonces tan solo se han apreciado cambios de tipo moderados ${ }^{60}$ al Código del Trabajo, cambios que a nuestro parecer no alteran de manera sustancial la regulación de las relaciones laborales.

c) El aspecto valorativo. - Este elemtoen se vincula con los valores individuales, trabajador en cuanto individuo, y con los valores colectivos, considerando al trabajador como parte de la sociedad ${ }^{61}$. El Código del Trabajo, luego de la reforma, considera que la huelga es un derecho que debe ser ejercido colectivamente por los trabajadores, según el inciso primero del artículo 345. Debemos entender, entonces, su reafirmación como derecho fundamental y su protección ${ }^{62}$. Sin embargo, llama la atención que durante la tramitación legislativa se debatiera en torno a la prohibición de remplazo (inciso del articulo 345) y en torno a la noción de adecuaciones necesarias. La prohibición quedo consagrada como un respaldo a la noción de huelga y las adecuaciones necesarias como facultad para el empleador para poder asegurar el derecho al trabajo de los trabajadores no sindicalizados ${ }^{63}$.

Se considera sólo la huelga dentro del procedimiento de negociación colectiva dejando un manto de dudas sobre si la concepción judicial ${ }^{64}$ que se había logrado será mantenida por la Corte Suprema.

Se ve una tendencia a privilegiar los derechos individuales por sobre los colectivos. Ello se ve reafirmado al proceder mediante esta reforma una nueva regulación de las limitaciones al derecho a huelga ${ }^{65}$. Se puede

${ }^{60}$ Así lo hemos calificado previamente, en Walker Errazuriz, Francisco, y Arellano Ortiz, Pablo, cit. (n. 46) p. 121.

${ }^{61}$ Walker Errazuriz, Francisco, y Arellano Ortiz, Pablo, cit. (n. 46) p. 32.

${ }^{62}$ Sobre la huelga como derecho fundamental véase GAMONAL CONTRERAS, Sergio, El derecho de huelga en la Constitución chilena, en Revista de Derecho, Universidad Católica del Norte (2013) vol. 20, n. 1, pp. 105- 127.

Cabe tener presente que en el seno de la Organización Internacional del Trabajo desde el año 2012 existe un fuerte debate acerca de si la huelga es o no un derecho fundamental contenido en los Convenios Nos.87 y 98, al respecto para interiorizarse sobre esta cuestión, véase BELLACE, Janice, La OIT y el derecho a huelga, en Revista Internacional del Trabajo, vol. 133 (2014), núm. 1, pp. 31-76.

${ }^{63}$ Artículo 403 del Código del Trabajo.

${ }^{64} \mathrm{Al}$ respecto ver las sentencias: Sentencia de la Corte Suprema, Cuarta Sala, 14 de enero de 2016, rol n ${ }^{\circ}$ 8.055-2015; Sentencia de la Corte Suprema, Cuarta Sala, 12 de mayo de 2015, rol no 15.293-2014, Sentencia de la Corte Suprema Cuarta Sala, 29 de enero de 2015, rol 10.444-2014; Sentencia de la Corte Suprema, Cuarta Sala, 4 de diciembre de 2014, rol 3.514-2.014; Sentencia de la Corte de Apelaciones de Santiago, 23 de octubre de 2015, rol n ${ }^{\circ}$ 1144-2015, y Sentencia de la Corte de Apelaciones de San Miguel, 9 de julio de 2014, rol n 183-2014.

${ }^{65} \mathrm{Al}$ respecto revisar los artículos 359 y siguientes del Código del Trabajo. 
notar un desincentivo a que los trabajadores ejerzan su legítimo derecho a la huelga. Considerándola como algo negativo y no como parte de un proceso normal de dialogo entre los actores sociales.

\section{REFLEXIONES FINALES}

De acuerdo con lo sostenido por Monereo y otros: "la complejidad del capitalismo avanzado y los procesos inducidos por la globalización económica, la pérdida del poder del Estado para gobernar la economía en el nuevo escenario, las transformaciones de las organizaciones productivas, la fragmentación de las clases trabajadoras o el debilitamiento del movimiento sindical, suponen un difícil reto para una construcción teorética que dé cuenta de la estructura y funcionamiento de las relaciones laborales (no sólo ya de amplio alcance, sino también para la teoría de alcance medio $)^{66}$.

Estas palabras recogen con precisión la encrucijada del sistema de relaciones laborales chileno. Una regulación liberal basada en el Plan Laboral ${ }^{67}$ que ha sufrido los embates de la globalización no ha logrado proyectarse hacia un modelo social en que sus valores tiendan a que la sociedad se articule y se produzca el bien común.

Hemos ya señalado a propósito de la reforma laboral "que se hace necesario que se reordene a mediano plazo la legislación hacia un equilibrio en las fuerzas de los actores, empleador y sindicato, ello con el fin de mejorar la acción de la huelga como un mecanismo efectivo de protección de la libertad sindical y como medio de corrección de las desigualdades sociales" 68

Una mirada a la regulación de los fenómenos laborales chilena bajo la óptica de la teoría de las relaciones laborales nos muestra que estamos lejos de un modelo óptimo. Los actores se encuentran en una posición de desequilibrio en donde los sindicatos han perdido poder frente a los grupos negociadores. La ideología del sistema sigue siendo la misma e incluso se ha vuelto más liberal que la existente. En lo valórico se puede constatar una disminución del valor de lo colectivo al darle más importancia al individuo por sobre la organización sindical y con una concepción de la huelga con limites más explícitos en cuanto a derecho fundamental.

Una revisión de la composición actual del sistema de relaciones laborales chileno a la luz de la postura de Dunlop da cuenta de un sistema aun en formación y con un fuerte contenido ideológico de corte liberal. Los objetivos trazados en la Agenda Laboral y en el proyecto de ley como

\footnotetext{
66 Monereo Pérez, José Luis et al., cit. (n. 18) p. 52 y 53.

67 Véase Narbona, Karina, cit. (n. 14); CaAmaño Rojo, Eduardo, cit. (n. 14).

68 Arellano Ortiz, Pablo et al., cit. (n. 9), p. 231.
} 
propósitos para reforzar las relaciones laborales no se ven totalmente cumplidos. Esto sin duda influye en no poder cumplir con los elementos de la teoría enunciada.

Es más, el objetivo enunciado en el discurso presidencial de 21 de mayo de 2014 de fortalecer la libertar sindical y sus 3 elementos a saber negociación colectiva, sindicatos y la huelga, no se ve alcanzado. Se ha sostenido que estos tres elementos deben estar en equilibrio formando un triángulo equilátero. Las modificaciones introducidas por la ley No 20.940 hacen que el triángulo en el caso chileno no sea equilátero, y además quede muy reducido en su tamaño. Con una huelga más limitada en su ejercicio, con más exigencias para formar sindicatos y con una excesiva reglamentación del procedimiento de negociación colectiva reglada.

Las críticas a esta nueva reglamentación también han provenido de la Organización Internacional del Trabajo.

Intentando responder a nuestra pregunta inicial de si la reforma de la ley No 20.940 nos lleva a pensar que existe un sistema de relaciones laborales en nuestro país, la respuesta luego de lo expuesto resulta ser negativa. Teniendo en cuenta las experiencias citadas, los Webbs y Dunlop, éstas parten de la base que la sociedad les entrega un lugar importante a los sindicatos y que éstos poseen la fuerza necesaria para desarrollar su accionar. Estos supuestos básicos de un sistema de relaciones laborales en nuestro país siguen sin cumplirse.

Compartimos lo expresado por el profesor Gamonal en este sentido señala que "Ojalá más pronto que tarde asumamos una consagración seria de la libertad sindical" ${ }^{69}$. Ante la falta de una consagración legal más consistente con el principio de la libertad sindical es de esperar que la jurisprudencia judicial que surja de los conflictos que se planteen, discuta y resuelva las cuestiones tratadas en este trabajo. Ya que hemos visto como la Corte Suprema se ha pronunciado acerca de la concepción de la huelga por lo que perfectamente podría, en un nuevo caso, darnos señales sobre cómo se están estructurando los elementos de nuestro sistema de relaciones laborales.

\section{BiBLIOGRAFÍA}

Aparicio Valdez, Luis y Bernedo Alvarado, Jorge, The Case for the Comparative and Interdisciplinary Study of Labour Relations, en BLANPAIN, Roger - BROMWICH, William; Rymkevich, Olga - Spattini, Silvia (eds.), Bulletin of Comparative Labour

69 Gamonal Contreras, Sergio, La reforma laboral: interpretación, antinomias y vacios, cit. (n. 14) p. 78. 
Relations, vol. 70, The Modernization of Labour Law and Industrial Relations in a Comparative Perspective, (Kluwer, 2009) p. 31.

Arellano Ortiz, Pablo; Liendo Roa, Ricardo y Walker Errázuriz, Francisco, Reforma Laboral ley No 20.940: Moderniza las relaciones laborales (Santiago, Librotecnia, 2016).

Arellano Ortiz, Pablo, Severin Concha, Juan Pablo y Feres Nazarala, María Ester (editores), Reforma al Derecho Colectivo del Trabajo: Examen critico de la ley $N^{o}$ 20.940 (Santiago, Thomson Reuters, 2016).

Bellace, Janice, La OIT y el derecho a huelga, en Revista Internacional del Trabajo, vol. 133 (2014), núm. 1, pp. 31-76.

Badinter Robert y LyOn-CAen, Antoine, Le travail et la loi (Fayard, Paris, 2015).

CAAmaño Rojo, Eduardo, Crítica a las ideas fundantes del actual modelo normativo de negociación colectiva en Chile y a su reforma, en Revista de Derecho de la Pontificia Universidad Católica de Valparaíso XLVI (2016) pp. 381 - 406.

Castro Sáez, Bernardo A., Aportes de Niklas Luhmann a la teoría de la complejidad, en Polis, Revista de la Universidad Bolivariana, vol. 10, No. 29 (2011), pp. 283-300.

Cole, Margaret, The Webbs and Social Theory, en The British Journal of Sociology, vol. 12, No. 2 (1961), pp. 93-105.

DAvIs, Stanley M., The Politics of Organizational Underdevelopment: Chile, en Industrial and Labour Relations Review, vol. 24, №. 1 (1970), pp. 73-83.

Derber, Milton, Divergent tendencies in industrial relations research, en Industrial and Labour Relations Review, vol. 17, No. 4 (1964), pp. 598-611.

Dunlop, John T. Sistema de relaciones industriales (Santiago, Editorial del Pacífico, 1967).

Gamonal Contreras, Sergio, La reforma laboral: interpretación, antinomias y vacios, en Revista Laboral Chilena (2016), pp. 73-78.

Gamonal Contreras, Sergio, La reforma laboral: un paso adelante y dos atrás, en Revista Laboral Chilena (2015), pp. 67-70.

Gamonal Contreras, Sergio, El derecho de huelga en la Constitución chilena, en Revista de Derecho, Universidad Católica del Norte (2013) vol. 20, n. 1, pp. 105- 127.

Kaufman, Bruce, Sidney and Beatrice Webb's Institutional Theory of Labor Markets and Wage Determination, Working Paper 2013-1-2, W. J. Usery Workplace Research Group Paper Series, Andrew Young School of Policy Studies, Georgia State University, January 2013.

KaUfMAN, Bruce, The Theoretical Foundation of Industrial Relations and its Implications for Labor Economics and Human Resource Management, en Industrial \& Labor Relations Review (2010) 64, p. 75.

KAUFMAN, Bruce, The global evolution of industrial relations: Events, ideas and the IIRA, (Geneva, International Labour Office, 2004).

KaUfman, Bruce, (ed) Theoretical perspectives on work and the employment relationship (Industrial Relations Research Association Series, Illinois, 2004).

KAUFMAN, Bruce, The origins \& evolution of the field of Industrial relations in the United States, en Cornell Studies in Industrial and Labor Relations, number 25, Ithaca and London, Cornell University Press, 1993.

Kerr, Clark - Dunlop, John T. - Harbison, Frederick - Myers, Charles A., Industrialism and Industrial Man (New York, Harvard University Press - Oxford University Press, 1960). 
Hyman, Richard, Tres hipótesis sobre el futuro de las relaciones laborales en Europa, en Revista Internacional del Trabajo, vol. 134 (2015), núm. 1, pp. 5-15.

Hyman, Richard, How Can We Study Industrial Relations Comparatively?, en BlanpaIn, Roger - Bromwich, William - Rymkevich, Olga \& Spattini, Silvia (eds.), Bulletin of Comparative Labour Relations, vol. 70, The Modernization of Labour Law and Industrial Relations in a Comparative Perspective, (Kluwer, 2009).

HyMAN, Richard, Relaciones industriales: una introducción marxista (Madrid, Blume, 1981).

Landsberger, Henry A. - Barrera, Manuel - Toro, Abel, The Chilean Labor Union Leader: A Preliminary Report on His Background and Attitudes, en Industrial and Labour Relations Review, vol. 17, №. 3 (1964), pp. 399-420.

LOKIEC, Pascal, Il faut sauver le droit du travail! (Paris, Odile Jacob, 2015).

LuCENA, Héctor, El enfoque de las relaciones industriales y los estudios laborales, en Enrique de la Garza Toledo (comp.), Los retos teóricos de los estudios del trabajo hacia el siglo XXI (Buenos Aires, Consejo Latinoamericano de Ciencias Sociales, 1999).

Marzi Muñoz, Daniela, Perspectiva de género, cuota en las directivas y nuevos objetos de negociación: un camino lento hacia el poder, en Arellano Ortiz, Pablo, SEverin Concha, Juan Pablo y Feres NAZARAla, María Ester (editores), Reforma al Derecho Colectivo del Trabajo, Examen crítico de la ley No 20.940 (Santiago, Thomson Reuters, 2016) pp. $63-86$.

Monereo Pérez, José Luis - Moreno Vida, María Nieves - Márquez Prieto, Antonio - Almendros González, Miguel Ángel - Díaz Aznarte, María Teresa - Fernández Avilés, José Antonio - Salas Porras, María, Manual de teoría de las relaciones laborales (Ed. Madrid, Tecnos, 2011).

Narbona, Karina, Antecedentes del modelo de relaciones laborales chileno (2a edición, Santiago, Fundación Sol, 2015).

Organización Internacional del Trabajo, Informe de la Comisión de Expertos en Aplicación de Convenios y Recomendaciones (artículos 19, 22, 23 y 35 de la Constitución), Tercer punto del orden del día: Informaciones y memorias sobre la aplicación de convenios y recomendaciones, Informe III (Parte 1A), Informe General y observaciones referidas a ciertos paises. Conferencia Internacional del Trabajo, 106 reunión, 2017 (Ginebra, Oficina Internacional del Trabajo, 2017).

Palomo Veliz, Rodrigo, El nuevo derecho de información de los sindicatos. Un ejemplo de reforma en la periferia del modelo, en Arellano Ortiz, Pablo, Severin Concha, Juan Pablo y Feres Nazarala, María Ester (editores): Reforma al Derecho Colectivo del Trabajo, Examen crítico de la ley No 20.940 (Santiago, Thomson Reuters, 2016) pp. $109-133$.

ReEd TrIPp, L. The Industrial Relations Discipline in American Universities, en Industrial and Labour Relations Review, vol. 17, No. 4 (1964), pp. 612-618.

RojAs MiÑo, Irene, Los pactos de adaptabilidad y el debate sobre su incorporación al sistema jurídico de relaciones laborales chileno, en Arellano OrTiz, Pablo, SEVErin Concha, Juan Pablo y Feres Nazarala, María Ester (editores) Reforma al Derecho Colectivo del Trabajo: Examen crítico de la ley No 20.940 (Thomson Reuters, Santiago, 2016), pp. $195-211$.

Thayer Arteaga, William y Novoa Fuenzalida, Patricio, Manual de Derecho del Trabajo (T. I, 2007; T. II, 2008; T. III, 2008 y T. IV 2010, Santiago, Editorial Jurídica de Chile). 
URTEAGA, Eguzkil La teoría de sistemas de Niklas Luhmann, en Contrastes. Revista Internacional de Filosofia, vol. XV (2010), pp. 301-317.

Walker Errazuriz, Francisco, y Arellano Ortiz, Pablo, Derecho de las relaciones laborales, Tomo 1, Derecho Individual del Trabajo (Santiago, Librotecnia, 2016).

Walker Errázuriz, Francisco, y Muga Naredo, José Antonio, Las relaciones industriales (Universidad de Chile, DERTO, 1978), pp. 44.

Webв, Sidney y Webв, Beatrice, Industrial Democracy (London, Longmans, Green, 1902).

Webb, Sidney y Webв, Beatrice, Industrial Democracy vol. I (London, Longmans, Green, 1897).

Webb, Sidney y WebB, Beatrice, Industrial Democracy vol. II (London, Longmans, Green, 1897).

Weвв, Sidney y Weвв, Beatrice, History of Trade Unionism (London, Amalgamated Society of Engineers, 1894). 